\section{Use of the National Early Warning Score for predicting in-hospital mortality in older adults admitted to the emergency department}

\author{
Inyong Kim, Hwan Song, Hyo Joon Kim, Kyu Nam Park, Soo Hyun Kim, \\ Sang Hoon Oh, Chun Song Youn
}

Department of Emergency Medicine, Seoul St. Mary Hospital, The Catholic University of Korea College of Medicine, Seoul, Korea

Objective The National Early Warning Score (NEWS), based on the patients' vital signs, detects clinical deterioration in critically ill patients and is used to reduce the incidence of in-hospital cardiac arrest. However, although mortality prediction based on vital signs may be difficult in older patients, the effectiveness of the NEWS has not yet been evaluated in this population. This study aimed to test the hypothesis that an elevated NEWS at admission increases the mortality risk in older patients admitted to the emergency department (ED).

Methods We conducted a single-center retrospective study, including patients admitted to the ED between November 2016 and February 2017. We included patients aged $>65$ years who were admitted to the ED for any medical problem. The NEWS was calculated at the time of ED admission. The primary outcome was in-hospital mortality.

Results In total, 3,169 patients were included in this study. Median age was 75 years (interquartile range [IOR], 70 to 80 years), and 1,557 (49.1\%) patients were male. The in-hospital mortality rate was 5.1\% (161 patients). Median NEWS was higher in non-survivors than in survivors (5 [IQR, 3-8] vs. 1 [IOR, 0-3], P<0.001). Multivariate logistic analysis showed that the NEWS was associated with in-hospital mortality, after adjusting for other confounders. The area under the curve of the NEWS for predicting in-hospital mortality was 0.820 (95\% confidence interval, 0.806 to 0.833$)$.

Conclusion Our results show that the NEWS at admission is associated with in-hospital mortality among patients aged $>65$ years.

Keywords Mortality; Aged; National Early Warning Score
eISSN: $2383-4625$

Received: 30 April 2019

Revised: 1 July 2019

Accepted: 9 July 2019

Correspondence to: Chun Song Youn Department of Emergency Medicine, Seoul St. Mary's Hospital, The Catholic University of Korea College of Medicine, 222 Banpo-daero, Seocho-gu,

Seoul 06591, Korea

E-mail:ycs1005@catholic.ac.kr ORCID

http://orcid.org/0000-0002-6709-7703

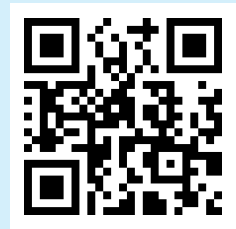

How to cite this article: Kim I, Song H, Kim HJ, Park KN, Kim SH, $\mathrm{Oh} \mathrm{SH}$, Youn CS. Use of the National Early Warning Score for predicting in-hospital mortality in older adults admitted to the emergency department. Clin Exp Emerg Med 2020;7(1):61-66.

This is an Open Access article distributed under the terms of the Creative Commons Attribution Non-Commercial License (https:// creativecommons.org/licenses/by-nc/4.0/). 


Capsule
Wummat is already known
The National Early Warning Score (NEWS) was originally developed to predict clinical deterioration, including cardiac
arrest within 24 hours, among ward patients, and it is known that the NEWS can predict in-hospital mortality in pa-
tients admitted to the emergency department.
What is new in the current study
$\begin{aligned} & \text { A high NEWS at admission is associated with all-cause in-hospital mortality among patients older than } 65 \text { years. A } \\ & \text { high NEWS for elderly patients in the emergency department can provide evidence to support decision-making pro- } \\ & \text { cesses related to hospital admissions, regardless of etiology. }\end{aligned}$

\section{INTRODUCTION}

Korea is becoming a super-aged society and is expected to have the fastest aging rate among the Organization for Economic Cooperation and Development countries. Aging is one of the leading causes of increased healthcare burden in emergency departments (EDs).' Older adults visit the ED more frequently than the younger. $^{2-4}$ Furthermore, accurate diagnosis and treatment may be delayed in elderly patients because they present with atypical signs and symptoms, use several medications, and have multiple comorbidities; these factors make mortality prediction be difficult.t ${ }^{5-7}$

The National Early Warning Score (NEWS) is a simple and rapid bedside tool involving seven physiologic parameters: respiration rate, oxygen saturation, use of supplemental oxygen, temperature, systolic blood pressure, heart rate, and consciousness level. ${ }^{8}$ It was originally developed to predict clinical deterioration, including cardiac arrest within 24 hours, in ward patients. Moreover, the NEWS is associated with mortality and intensive care unit admission in patients admitted to the ED. ${ }^{9-12}$ As mentioned above, the NEWS is based on the patient's vital signs. However, in elderly patients, mortality prediction based on vital signs may be difficult because older patients may show subtle changes in vital signs due to a reduced physiological response to physiological stress. ${ }^{13,14}$ Despite this difficulty, few studies have evaluated the effectiveness of the NEWS in elderly patients admitted to the ED.

The aim of this study was to test the hypothesis that an elevated NEWS at admission increases the risk of mortality in older patients admitted to the ED. We assessed the association between the NEWS at admission and in-hospital mortality in older patients admitted to the ED. To the best of our knowledge, this is the first report to describe the association between the NEWS and in-hospital mortality among older patients.

\section{METHODS}

\section{Patients and setting}

We conducted a retrospective observational study of consecutive patients admitted to the ED of a single academic tertiary hospital between November 2016 and February 2017. This study was approved by the institutional review board of Seoul St. Mary's Hospital (file number: XC17REDI0071), and the requirement for informed consent was waived because of the study's retrospective nature.

We included patients aged $>65$ years admitted to the ED for any medical issue. The following patients were excluded: patients with trauma-related injuries; those visiting the ED for a nonmedical issue; and those who died before arrival to the hospital. The primary outcome of this study was in-hospital mortality.

\section{Data collection}

Data of the following demographic characteristics were collected from patients' medical records: age, sex, and comorbidities, including diabetes mellitus, hypertension, coronary artery disease, liver cirrhosis, end-stage renal disease (receiving renal replacement therapy), chronic lung disease, congestive heart failure, and malignancy. We also collected data of the following clinical findings: systolic blood pressure, diastolic blood pressure, mean arterial pressure, heart rate, respiratory rate, body temperature, mental status, and Korean Triage and Acuity Scale (KTAS) score. The KTAS is a triage tool consisting of five levels for classification of patients according to their chief complaints and vital signs. ${ }^{15}$

\section{The NEWS}

The NEWS consists of seven physiological variables: systolic blood pressure, heart rate, respiratory rate, body temperature, oxygen saturation, use of supplemental oxygen, and level of consciousness (Table 1). ${ }^{8}$ The NEWS is calculated by summing the scores 
Table 1. The National Early Warning Score

\begin{tabular}{|c|c|c|c|c|c|c|c|}
\hline Physiological parameters & 3 & 2 & 1 & 0 & 1 & 2 & 3 \\
\hline Respiration rate (BPM) & $\leq 8$ & & $9-11$ & $12-20$ & & $21-24$ & $\geq 25$ \\
\hline Oxygen saturation (\%) & $\leq 91$ & $92-93$ & $94-95$ & $\geq 96$ & & & \\
\hline Any supplemental oxygen & & Yes & & No & & & \\
\hline Temperature $\left({ }^{\circ} \mathrm{C}\right)$ & $\leq 35.0$ & & $35.1-36.0$ & $36.1-38.0$ & $38.1-39.0$ & $\geq 39.1$ & \\
\hline $\mathrm{SBP}(\mathrm{mmHg})$ & $\leq 90$ & $91-100$ & $101-110$ & $111-219$ & & & $\geq 220$ \\
\hline Heart rate (BPM) & $\leq 40$ & & $41-50$ & $51-90$ & $91-110$ & $110-130$ & $\geq 131$ \\
\hline Consciousness level & & & & A & & & $V, P$, or $U$ \\
\hline
\end{tabular}

$S B P$, systolic blood pressure; $A$, alert; $V$, verbal response; $P$, pain response; $U$, unresponsive.

Table 2. Baseline characteristics in patients with or without in-hospital mortality

\begin{tabular}{|c|c|c|c|}
\hline & $\begin{array}{l}\text { Survivors } \\
(n=3,008)\end{array}$ & $\begin{array}{c}\text { Non-survivors } \\
\quad(n=161)\end{array}$ & P-value \\
\hline Age & $75(70-80)$ & 77 (70-83) & 0.009 \\
\hline Sex, male & $1,453(48.3)$ & $104(64.6)$ & $<0.001$ \\
\hline \multicolumn{4}{|l|}{ Past history } \\
\hline Diabetes mellitus & 849 (28.2) & $59(36.6)$ & 0.021 \\
\hline Hypertension & $1,611(53.6)$ & $87(54.0)$ & 0.905 \\
\hline CAD & 457 (15.2) & $24(14.9)$ & 0.922 \\
\hline Liver cirrhosis & $66(2.2)$ & $7(4.3)$ & 0.076 \\
\hline ESRD on RRT & $87(2.9)$ & $8(5.0)$ & 0.132 \\
\hline Chronic lung disease & $503(16.7)$ & $53(32.9)$ & $<0.001$ \\
\hline $\mathrm{CHF}$ & $57(1.9)$ & $6(3.7)$ & 0.105 \\
\hline Malignancy & $755(25.1)$ & $86(53.4)$ & $<0.001$ \\
\hline \multicolumn{4}{|l|}{ Vital signs } \\
\hline $\mathrm{SBP}(\mathrm{mmHg})$ & $140(122-155)$ & $125(109.5-139.5)$ & $<0.001$ \\
\hline $\mathrm{DBP}(\mathrm{mmHg})$ & 78 (68-89) & $70(60-84)$ & $<0.001$ \\
\hline $\mathrm{MAP}(\mathrm{mmHg})$ & $99(87-110)$ & $90(78-100)$ & $<0.001$ \\
\hline Heart rate (BPM) & 85 (74-98) & $98(85-115)$ & $<0.001$ \\
\hline Respiration rate (BPM) & $19(18-20)$ & $20(18-22)$ & $<0.001$ \\
\hline Temperature $\left({ }^{\circ} \mathrm{C}\right)$ & $36.8(36.5-37.2)$ & $37.0(36.5-37.7)$ & 0.107 \\
\hline Mental status & & & $<0.001$ \\
\hline Alert & $2,900(96.4)$ & $129(80.1)$ & \\
\hline Verbal & $41(1.4)$ & $8(5.0)$ & \\
\hline Pain & $61(2.0)$ & 18 (11.2) & \\
\hline Unresponsive & $6(0.2)$ & $6(3.7)$ & \\
\hline Triage level & & & $<0.001$ \\
\hline Immediate & $23(0.8)$ & $5(3.1)$ & \\
\hline Emergent & 299 (9.9) & $46(28.6)$ & \\
\hline Urgent & $2,050(68.2)$ & $105(65.2)$ & \\
\hline Semiurgent & 537 (17.9) & $4(2.5)$ & \\
\hline Nonurgent & 99 (3.3) & $1(0.6)$ & \\
\hline NEWS & $1(0-3)$ & $5(3-8)$ & $<0.001$ \\
\hline Aggregated NEWS & & & $<0.001$ \\
\hline Aggregate 0-4 & $2,655(88.3)$ & $73(45.3)$ & \\
\hline Aggregate 5-6 & $231(7.7)$ & 35 (21.7) & \\
\hline Aggregate $\geq 7$ & $122(4.1)$ & 53 (32.9) & \\
\hline ICU admission & 269 (8.9) & $74(46.0)$ & $<0.001$ \\
\hline Hospital admission ${ }^{\text {a) }}$ & $1,255(41.7)$ & 150 (93.2) & $<0.001$ \\
\hline
\end{tabular}

Variables are expressed as median (interquartile range) or $n(\%)$.

$C A D$, coronary artery disease; ESRD, end-stage renal disease; RRT, renal replace-

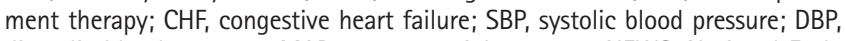
diastolic blood pressure; MAP, mean arterial pressure; NEWS, National Early Warning Score; ICU, intensive care unit.

a) Hospital admission includes both ward admission and ICU admission.
Table 3. The association between KTAS and NEWS

\begin{tabular}{lccccc}
\hline & $\begin{array}{c}\text { KTAS 1 } \\
(n=28)\end{array}$ & $\begin{array}{c}\text { KTAS 2 } \\
(n=345)\end{array}$ & $\begin{array}{c}\text { KTAS 3 } \\
(n=2,155)\end{array}$ & $\begin{array}{c}\text { KTAS 4 } \\
(n=541)\end{array}$ & $\begin{array}{c}\text { KTAS 5 } \\
(n=100)\end{array}$ \\
\hline NEWS & & & & & \\
Aggregate 0-4 & $13(46.4)$ & $236(68.4)$ & $1,848(85.8)$ & $532(98.3)$ & $99(99.0)$ \\
Aggregate 5-6 & $3(10.7)$ & $38(11.0)$ & $216(10.0)$ & $8(1.5)$ & $1(1.0)$ \\
Aggregate $\geq 7$ & $12(42.9)$ & $71(20.6)$ & $91(4.2)$ & $1(0.2)$ & $0(0)$ \\
\hline
\end{tabular}

KTAS, Korean Triage and Acuity Scale; NEWS, National Early Warning Score.

for each of the seven variables (0-3 points). NEWS-based categories were classified as low risk (0-4), intermediate risk (5-6), and high risk ( $\geq 7$ ). The NEWS was calculated at the time of ED admission.

\section{Statistical analysis}

We presented non-normally distributed continuous data as medians and interquartile ranges (IQRs) and presented categorical data as percentages. To compare patient characteristics between groups, we used the Mann-Whitney U-test (for continuous variables) and the Fisher exact test and chi-squared test (for categorical variables). Univariate analysis was performed to determine the covariates for in-hospital mortality. Multivariate logistic regression analysis was performed to select covariates. Variables with P-values $<0.05$ in univariate analysis were selected for multivariate analysis.

To evaluate the association between the NEWS and in-hospital mortality, the NEWS were aggregated into tertiles using the following cutoff values: $0-4,5-6$, and $\geq 7$. The NEWS was also examined as a continuous variable. Statistical analyses were performed using IBM SPSS Statistics ver. 24.0 (IBM Corp., Armonk, NY, USA) and MedCalc ver. 15.2.2 (MedCalc Software, Mariakerke, Belgium). P-values $\leq 0.05$ were considered statistically significant.

\section{RESULTS}

\section{Characteristics of the study population}

In total, 3,169 patients were included in the analysis. Median age 
was 75 years (IQR, 70 to 80 years), and 1,557 (49.1\%) patients were male. The in-hospital mortality rate was $5.1 \%(n=161)$. The baseline characteristics of the study population are presented in Table 2. Median NEWS was 1.0 (IOR, 0 to 3.0). The NEWS was

Table 4. Odds ratios for all-cause in-hospital mortality events

\begin{tabular}{|c|c|c|c|}
\hline \multirow{2}{*}{ Variable } & \multicolumn{3}{|c|}{ Univariate } \\
\hline & Odds ratio & $95 \% \mathrm{Cl}$ & P-value \\
\hline Age & 1.032 & $1.010-1.053$ & 0.004 \\
\hline Sex, male & 1.953 & $1.403-2.718$ & $<0.001$ \\
\hline Diabetes mellitus & 1.471 & $1.057-2.047$ & 0.022 \\
\hline Hypertension & 1.020 & $0.742-1.401$ & 0.905 \\
\hline CAD & 0.978 & $0.627-1.526$ & 0.922 \\
\hline Liver cirrhosis & 2.026 & $0.914-4.490$ & 0.082 \\
\hline ESRD on RRT & 1.756 & $0.836-3.687$ & 0.137 \\
\hline Chronic lung disease & 2.444 & $1.735-3.442$ & $<0.001$ \\
\hline CHF & 2.004 & $0.851-4.720$ & 0.112 \\
\hline Malignancy & 3.422 & $2.484-4.714$ & $<0.001$ \\
\hline SBP & 0.978 & $0.972-0.984$ & $<0.001$ \\
\hline DBP & 0.977 & $0.967-0.986$ & $<0.001$ \\
\hline MAP & 0.973 & $0.964-0.982$ & $<0.001$ \\
\hline Heart rate & 1.029 & $1.021-1.037$ & $<0.001$ \\
\hline Respiratory rate & 1.118 & $1.069-1.168$ & $<0.001$ \\
\hline Temperature & 1.206 & $1.013-1.435$ & 0.035 \\
\hline \multicolumn{4}{|l|}{ Mental status } \\
\hline Alert & Reference & Reference & Reference \\
\hline Verbal & 4.386 & $2.015-9.548$ & $<0.001$ \\
\hline Pain & 6.634 & $3.810-11.550$ & $<0.001$ \\
\hline Unresponsive & 22.481 & 7.152-70.661 & $<0.001$ \\
\hline \multicolumn{4}{|l|}{ Triage level } \\
\hline Immediate & 21.522 & 2.398-193.157 & 0.006 \\
\hline Emergent & 15.231 & $2.073-111.884$ & 0.007 \\
\hline Urgent & 5.071 & $0.700-36.710$ & 0.108 \\
\hline Semiurgent & 0.737 & $0.082-6.667$ & 0.786 \\
\hline Nonurgent & Reference & Reference & Reference \\
\hline NEWS & 1.485 & $1.410-1.565$ & $<0.001$ \\
\hline \multicolumn{4}{|l|}{ Aggregated NEWS } \\
\hline Aggregate 0-4 & Reference & Reference & \\
\hline Aggregate 5-6 & 5.511 & $3.603-8.427$ & $<0.001$ \\
\hline Aggregate $\geq 7$ & 15.800 & $10.617-23.513$ & $<0.001$ \\
\hline
\end{tabular}

$\mathrm{Cl}$, confidence interval; $\mathrm{CAD}$, coronary artery disease; $E S R D$, end-stage renal disease; RRT, renal replacement therapy; $C H F$, congestive heart failure; SBP, systolic blood pressure; DBP, diastolic blood pressure; MAP, mean arterial pressure; NEWS, National Early Warning Score. higher in non-survivors (median, 5; IQR, 3 to 8) than in survivors (median, $1 ; \mathrm{IQR}, 0$ to $3, \mathrm{P}<0.001$ ). The associations between the KTAS and NEWS are presented in Table 3.

\section{Logistic regression analysis}

On univariate analysis, age, male sex, diabetes mellitus, liver cirrhosis, end-stage renal disease on renal replacement therapy, chronic lung disease, congestive heart failure, malignancy, and triage level were found to be associated with in-hospital mortality and were selected as covariates (Table 4). The NEWS was examined as a continuous and categorical variable. Both models showed an association between the NEWS and in-hospital mortality. When the NEWS was examined as a continuous variable, the adjusted odds ratio for in-hospital mortality was 1.475 (95\% confidence interval [CI], 1.395 to 1.561). When the NEWS was examined as a categorical variable, the adjusted odds ratios for in-hospital mortality were as follows: NEWS 5-6, $4.666(95 \% \mathrm{Cl}$, 2.986 to 7.292$)$ and NEWS $\geq 7,13.641$ (95\% Cl, 8.899 to 20.909) (Table 5).

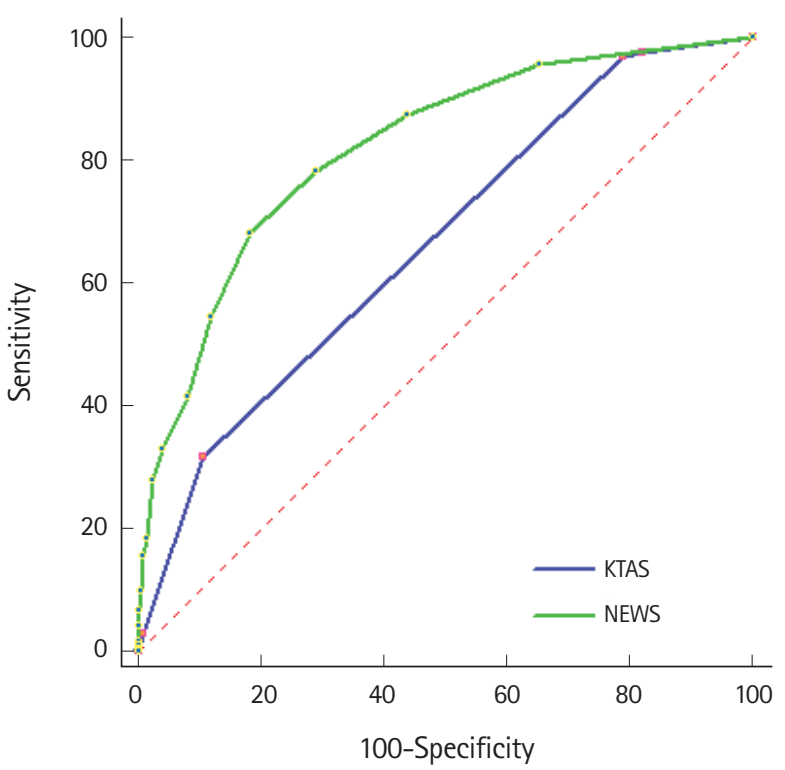

Fig. 1. Comparison of ROC curve for predicting in-hospital mortality. AUC for KTAS: 0.664 (95\% Cl, 0.647-0.680), for NEWS: $0.820(95 \% \mathrm{Cl}$, $0.806-0.833(p<0.001))$

Table 5. ORs for NEWS in the prediction of all-cause in-hospital mortality

\begin{tabular}{|c|c|c|c|c|}
\hline & \multicolumn{3}{|c|}{ Aggregated NEWS } & \multirow{2}{*}{$\begin{array}{l}\text { NEWS as continuous variable } \\
\text { OR }(95 \% \mathrm{Cl})\end{array}$} \\
\hline & NEWS $0-4(n=2,739$, death $=73)$ & NEWS 5-6 $(n=268$, death $=35)$ & NEWS $\geq 7(n=176$, death $=54)$ & \\
\hline Model $1^{\text {a) }}$ & (Reference) & 4.666 (2.986-7.292) & 13.641 (8.899-20.909) & $1.475(1.395-1.561)$ \\
\hline
\end{tabular}

$\mathrm{OR}$, odds ratio; NEWS, National Early Warning Score; $\mathrm{Cl}$, confidence interval.

${ }^{a)} \mathrm{Age}+\mathrm{sex}+$ diabetes mellitus+liver cirrhosis+end-stage renal disease on renal replacement therapy+chronic lung disease+congestive heart failure+malignancy+KTAS level. 


\section{Prognostic value of the NEWS}

The area under the curve of the NEWS for predicting in-hospital mortality was $0.820(95 \% \mathrm{Cl}, 0.806$ to 0.833$)$ (Fig. 1).

\section{DISCUSSION}

This study aimed to assess whether the NEWS at admission was associated with in-hospital mortality in older patients admitted to the ED. Our analysis showed that the NEWS at the time of ED admission was significantly correlated with in-hospital mortality among patients aged $>65$ years. When the NEWS increased by 1 , the in-hospital mortality rate increased by $47.5 \%$. If the patient had a NEWS of $\geq 7$, the risk of in-hospital death was $30.3 \%$. However, if the patient had a NEWS of $0-4$, the risk of in-hospital death was $2.7 \%$. To the best of our knowledge, this is the first report to describe the association between the NEWS and in-hospital mortality among older patients.

The triage of patients on arrival at the ED is very important for appropriate patient care. However, accurate patient triage requires experience and may be affected by interobserver variability. ${ }^{16,17}$ Several triage tools have been developed for use in the ED, including the KTAS, which is now being used in clinical practice. ${ }^{18,19}$ In this study, the NEWS was associated with in-hospital mortality in older adults admitted to the ED. Because the NEWS is an objective tool, additional use of the NEWS for patient triage could be considered. However, this should be studied further.

Owing to the increasing elderly population, physicians should give more medical attention to elderly patients., ${ }^{1,2}$ Older adults visit the ED more frequently than the younger. Predicting mortality and performing appropriate triage may be difficult in older patients because they present atypical signs and symptoms, have multiple comorbidities, and use multiple medications. ${ }^{5-7}$ However, risk factors for in-hospital mortality in older adults admitted to the ED have rarely been investigated. One study proposed a strategy based on targeting high-risk patients. ${ }^{4}$ The results of the current study may also assist in the identification of older patients with a high risk of in-hospital mortality.

Churpek et al. reported that vital signs detect cardiac arrest more accurately in nonelderly ward patients (area under the receiver operating characteristic curve, $0.85 ; 95 \% \mathrm{Cl}, 0.82$ to 0.88 ) than in elderly ward patients (area under the receiver operating characteristic curve, $0.71 ; 95 \% \mathrm{Cl}, 0.68$ to 0.75$).{ }^{20}$ In addition, they suggested the need for more accurate methods of risk stratification, such as stratification by comorbidities. However, considering this study only included elderly patients, no comparison was made with younger adults. Some studies have reported that the predictive ability of the NEWS increases on combining several lab- oratory results with the NEWS., ${ }^{90-22}$ This combination method should also be validated in older patients to improve predictive ability in this patient group.

This study had several limitations. First, this was a single-center, retrospective observational study. The baseline characteristics of ED patients may vary according to region and country. Therefore, the generalizability of the results of this study may be limited. Second, there could be unknown confounding factors due to the retrospective nature of this study. Moreover, we did not analyze laboratory or imaging findings, which indicates the need for prospective multicenter studies in the future. Third, the primary outcome of this study was all-cause in-hospital mortality, and we did not evaluate the association between the NEWS and cause-specific mortality. Finally, this study had a relatively short study period. Therefore, seasonal variations in the patient population should be considered while interpreting our results.

In conclusion, the NEWS is a simple and rapid bedside tool for predicting in-hospital mortality among patients aged $>65$ years. A high NEWS in elderly patients admitted to the ED may provide evidence to support decision-making processes related to hospital admission, regardless of etiology.

\section{CONFLICT OF INTEREST}

No potential conflict of interest relevant to this article was reported.

\section{REFERENCES}

1. Organization for Economic Cooperation and Development. OECD economic surveys: Korea. Paris: Organization for Economic Cooperation and Development; 2016.

2. Mion LC, Palmer RM, Anetzberger GJ, Meldon SW. Establishing a case-finding and referral system for at-risk older individuals in the emergency department setting: the SIGNET model. J Am Geriatr Soc 2001;49:1379-86.

3. Roberts DC, McKay MP, Shaffer A. Increasing rates of emergency department visits for elderly patients in the United States, 1993 to 2003. Ann Emerg Med 2008;51:769-74.

4. Samaras N, Chevalley T, Samaras D, Gold G. Older patients in the emergency department: a review. Ann Emerg Med 2010; 56:261-9.

5. Aminzadeh F, Dalziel WB. Older adults in the emergency department: a systematic review of patterns of use, adverse outcomes, and effectiveness of interventions. Ann Emerg Med 2002;39:238-47.

6. Singal BM, Hedges JR, Rousseau EW, et al. Geriatric patient 
emergency visits. Part I: Comparison of visits by geriatric and younger patients. Ann Emerg Med 1992;21:802-7.

7. Strange GR, Chen EH. Use of emergency departments by elder patients: a five-year follow-up study. Acad Emerg Med 1998; 5:1157-62.

8. Smith GB, Prytherch DR, Meredith P, Schmidt PE, Featherstone PI. The ability of the National Early Warning Score (NEWS) to discriminate patients at risk of early cardiac arrest, unanticipated intensive care unit admission, and death. Resuscitation 2013;84:465-70.

9. Jarvis SW, Kovacs C, Badriyah T, et al. Development and validation of a decision tree early warning score based on routine laboratory test results for the discrimination of hospital mortality in emergency medical admissions. Resuscitation 2013; 84:1494-9.

10. Jo $S$, Yoon J, Lee JB, Jin Y, Jeong T, Park B. Predictive value of the National Early Warning Score-Lactate for mortality and the need for critical care among general emergency department patients. J Crit Care 2016;36:60-8.

11. Kivipuro M, Tirkkonen J, Kontula $T$, et al. National early warning score (NEWS) in a Finnish multidisciplinary emergency department and direct vs. late admission to intensive care. Resuscitation 2018;128:164-9.

12. Alam N, Vegting IL, Houben $E$, et al. Exploring the performance of the National Early Warning Score (NEWS) in a European emergency department. Resuscitation 2015;90:111-5.

13. Boss GR, Seegmiller JE. Age-related physiological changes and their clinical significance. West J Med 1981;135:434-40.

14. Chester JG, Rudolph JL. Vital signs in older patients: age-related changes. J Am Med Dir Assoc 2011;12:337-43.
15. Kwon H, Kim YJ, Jo YH, et al. The Korean Triage and Acuity Scale: associations with admission, disposition, mortality and length of stay in the emergency department. Int J Qual Health Care 2019;31:449-55.

16. Eitel DR, Travers DA, Rosenau AM, Gilboy N, Wuerz RC. The emergency severity index triage algorithm version 2 is reliable and valid. Acad Emerg Med 2003;10:1070-80.

17. Manos D, Petrie DA, Beveridge RC, Walter S, Ducharme J. Inter-observer agreement using the Canadian Emergency Department Triage and Acuity Scale. CJEM 2002;4:16-22.

18. Storm-Versloot MN, Ubbink DT, Chin a Choi V, Luitse JS. Observer agreement of the Manchester Triage System and the Emergency Severity Index: a simulation study. Emerg Med J 2009;26:556-60.

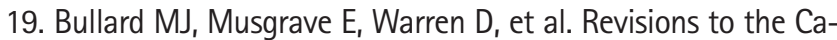
nadian Emergency Department Triage and Acuity Scale (CTAS) Guidelines 2016. CJEM 2017;19:S18-27.

20. Churpek MM, Yuen TC, Winslow C, Hall J, Edelson DP. Differences in vital signs between elderly and nonelderly patients prior to ward cardiac arrest. Crit Care Med 2015;43:816-22.

21. Eckart $A$, Hauser $\mathrm{SI}$, Kutz $A$, et al. Combination of the National Early Warning Score (NEWS) and inflammatory biomarkers for early risk stratification in emergency department patients: results of a multinational, observational study. BMJ Open 2019; 9:e024636.

22. Rasmussen $\sqcup H$, Ladelund $\mathrm{S}$, Haupt TH, Ellekilde GE, EugenOlsen J, Andersen 0 . Combining national early warning score with soluble urokinase plasminogen activator receptor (suPAR) improves risk prediction in acute medical patients: a registry-based cohort study. Crit Care Med 2018;46:1961-8. 